\title{
Towards a full understanding of the growth dynamics and optical response of self-assembled photonic colloidal crystal films
}

\author{
Gabriel S. Lozano, ${ }^{a}$ Luis A. Dorado, ${ }^{b}$ Ricardo A. Depine ${ }^{b}$ and Hernán Míguez ${ }^{* a}$ \\ DOI: $10.1039 / \mathrm{b} 811955 \mathrm{~d}$ \\ Recent advances in the comprehension of the growth dynamics of colloidal crystal films opens \\ the door to rational design of experiments aiming at fabricating lattices in which the density of \\ intrinsic defects is minimized. Since such imperfections have a dramatic effect on scattered \\ light of wavelength smaller than the lattice constant, the evaluation of the experimental \\ optical response at those energy ranges, based on the comparison to rigorous calculations, is \\ identified as the most sensitive guide to accurately evaluate the progress towards the actual \\ realization of defect-free colloidal crystals.
}

${ }^{a}$ Instituto de Ciencia de Materiales de Sevilla, Consejo Superior de Investigaciones Cientificas, Sevilla,Spain.E-mail: hernan@icmse.csic.es ${ }^{b}$ Grupo de Electromagnetismo Aplicado,

Departamento de Física, Facultad de Ciencias Exactas y Naturales, Universidad de Buenos Aires, Buenos Aires, Argentina

\section{Introduction}

Among the many methods developed in recent years to form colloidal lattices that exhibit photonic crystal properties, those based on evaporation-induced selfassembly onto vertical or tilted substrates have become the most widely employed today. The advent of this method ${ }^{1}$ and its later improvements ${ }^{2}$ has allowed the realization of crystalline films of unprecedented structural quality. For the first time, a number of optical features, which were obscured in similar lattices with

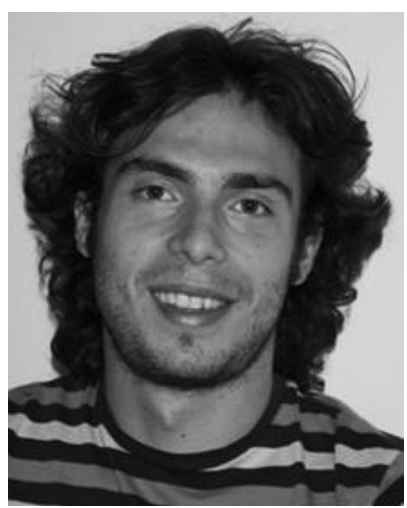

Gabriel S. Lozano

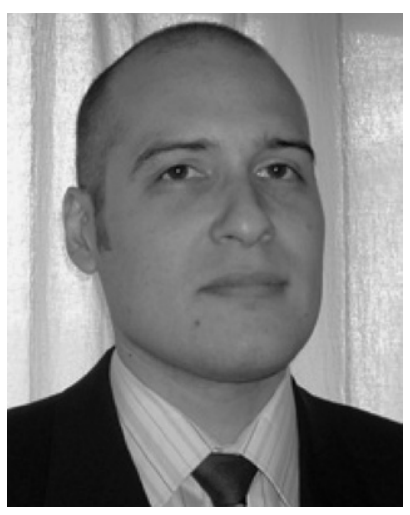

Luis A. Dorado
Gabriel S. Lozano (Córdoba, Spain, 1983) received his Physics Degree from the Cordoba University in 2006. He is a second year PhD student in Miguez's group. His thesis work is focused on the analysis of both the mechanism of crystallization of microspheres through selfassembly and the optical response of the resulting photonic crystals in the high energy range.

Luis A. Dorado (Tucumán, Argentina 1976) received his Electronic Engineering Degree from the National University of Tucumán, Argentina, in 2001. He teaches Antennas at the National Technological University in Buenos Aires. He has developed commercial and academic simulation software for computing radiation and scattering from complex photonic materials.

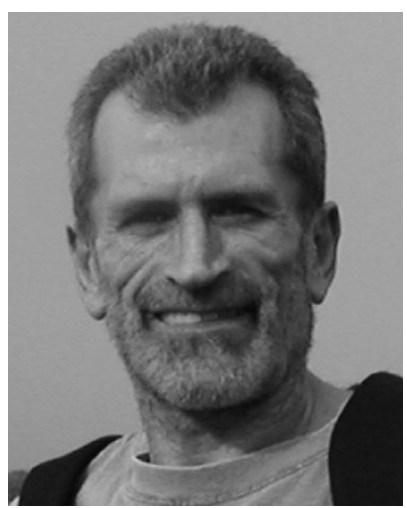

Ricardo A. Depine

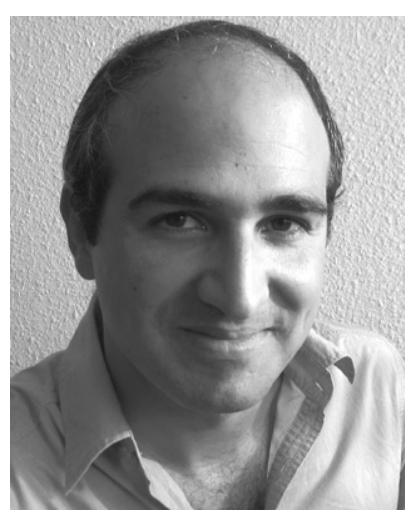

Hernán Míguez
Ricardo A. Depine was born in Buenos Aires, Argentina. Presently, he is a Professor in the Departamento de Física, Facultad de Ciencias Exactas y Naturales, Universidad de Buenos Aires, where he teaches electromagnetic theory and optics. He is also a Researcher of the Consejo Nacional de Investigaciones Cientificas y Técnicas (CONICET). His current research interests lie in the field of electromagnetism of complex media and nanotechnology.

Hernán Míguez (Buenos Aires, Argentina, 1971) is a tenured researcher of the Spanish Research Council (CSIC) and leads the group of Optical Nanomaterials in the Institute of Materials Science of Seville. His group's activities are devoted to the development, characterization and modeling of new photonic architectures for applications in different fields. 
a lower degree of order, were observable and measurable. Optical diffraction, ${ }^{3}$ slow light propagation at stop band edges, ${ }^{4}$ anomalous light speed at higher energy ranges, ${ }^{5}$ surface resonant states, ${ }^{6,7}$ and intensity modulations in the spectra of backwards reflected, forwardly transmitted $^{8-10}$ or diffracted beams ${ }^{11}$ are some of them. In spite of the importance that this type of film has acquired in the materials physics and chemistry community, and their interesting potential applications, the mechanism by which these face-centred-cubic lattices are actually formed has remained unknown and has been the subject of much speculation. ${ }^{12,13}$ In addition, as the crystal quality is improved, an exciting debate has started about the origin of the optical properties now observable in ranges for which photon wavelengths are on the same order or smaller than the lattice constant. ${ }^{14-17}$

In this highlight, we will overview some recent developments in the understanding of the growth dynamics of colloidal crystal films, which have provided an explanation for a number of reported observations whose cause remained unclear. Although a full description of the crystallization process is yet to be achieved, this achievement might open a way to the rational design of less defective crystals. On a closely related note, we will present a description of the effect that intrinsic imperfections have on light incident on a colloidal crystal film, putting special emphasis on the response at wavelengths shorter than the lattice constant of the crystal. We seek to identify the main challenges that must be faced to achieve and evaluate highly ordered colloidal photonic crystal lattices.

\section{Growth dynamics of colloidal crystal films}

The mechanism by which a colloidal crystal film is formed onto a substrate placed in a non-horizontal position within a beaker containing a dispersion of monodisperse microspheres involves two different aspects. On the one hand, a solid film is deposited onto the substrate as a result of the evaporation of the liquid phase of the particle suspension. This process takes place at the contact line between the meniscus and the substrate and, in a first approximation, is indepen-

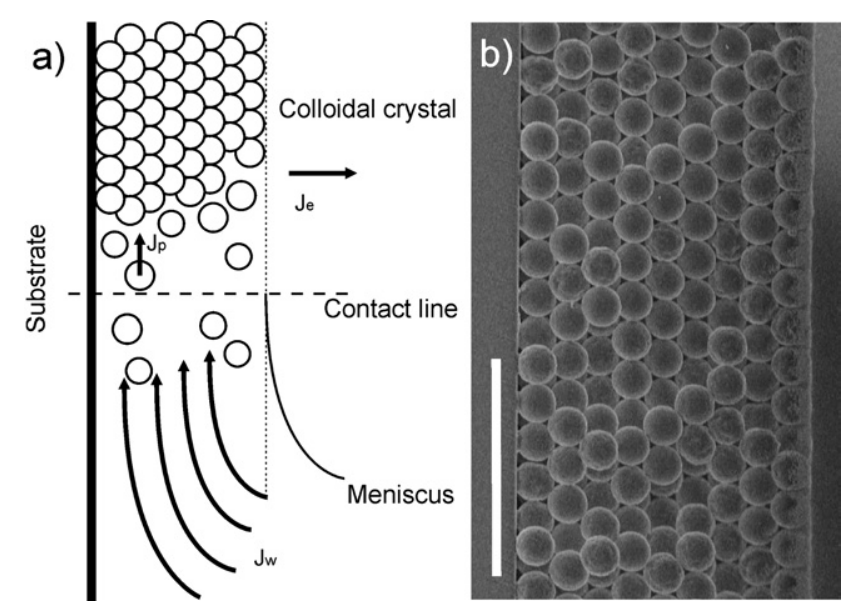

Fig. 1 (a) Scheme of the particle and water fluxes in the vicinity of the forming lattice in the meniscus. (b) SEM micrograph of the cross-section along a horizontal line, perpendicular to the growth direction of a colloidal crystal vertically deposited from a suspension of polystyrene latex spheres (average diameter $0.75 \mu \mathrm{m}$ ). Scale bar is $4 \mu \mathrm{m}$.

dent of the type of packing (random or ordered) of the particles present in the film. On the other hand, under specific conditions, the spheres in the film tend to self-organize, typically in a face-centredcubic (fcc) structure. Fig. 1 shows a scheme of the formation of a crystalline colloidal particle film onto a planar substrate as well as a scanning electron microscopy image of a cross-section of one such film from which the fcc ordering can be inferred. At present, a comprehensive model that takes into account both processes, i.e., film deposition and crystallization, is missing and this has prevented the rational design of the film growth. Attempts to improve crystalline quality and thus optical properties have been based on trial-and-error approaches.

We have recently shown that some important structural features of colloidal crystal films can be understood using a model that describes, in terms of the experimental variables, the deposition of the colloidal particle film onto a vertical or tilted flat substrate. ${ }^{18,19}$ To develop our model, we adapted deposition models previously developed to understand the structural properties of films formed from the drying of colloidal suspension drops on flat substrates. ${ }^{20}$ From our model, whose full details can be found in ref. 18, we could extract detailed information of the film deposition dynamics that allowed us to propose, for the first time, an equation for the film formation speed at the contact line between the substrate and the suspension meniscus. This crystalline film formation speed $v_{\mathrm{g}}(t)$ oscillates and decays with time following the expression:

$$
v_{\mathrm{g}}(t) \simeq V_{\mathrm{S}}\left[1-\mathrm{e}^{-\lambda t} \cos (\mu t)\right]
$$

In which $V_{\mathrm{s}}$ is the speed of the contact line attained when the steady state is reached $(t \rightarrow \infty)$, and $\mu$ and $\lambda$, the period and the damping parameter of the periodic fluctuations respectively, are functions of the experimental parameters of the deposition process. On the other hand, crystal thickness $H(t)$ can be approximated by the expression

$$
H(t)=C_{1} t+\frac{C_{2}}{v_{\mathrm{g}}(t)}
$$

Where $C_{1}$ and $C_{2}$ are also time-independent functions of the deposition parameters. Combination of eqns (1) and (2) yields an interesting prediction: Crystalline film thickness should oscillate with time during the deposition process. Hence, a periodic profile (or, as particular case, a step-like profile, as shown in ref. 18) should be observable along the growth direction of the film on the substrate as a direct consequence of the stick-slip character of the film formation. Microspectroscopy analysis of the optical reflectance of very small areas $\left(100 \mu \mathrm{m}^{2}\right)$, taken on numerous regularly spaced spots on the film surface, provided the first experimental evidence of such thickness fluctuations. An example of one of the spectra used for such analysis is presented in Fig. 2(a) (red solid line). The maximum detected is the result of the 

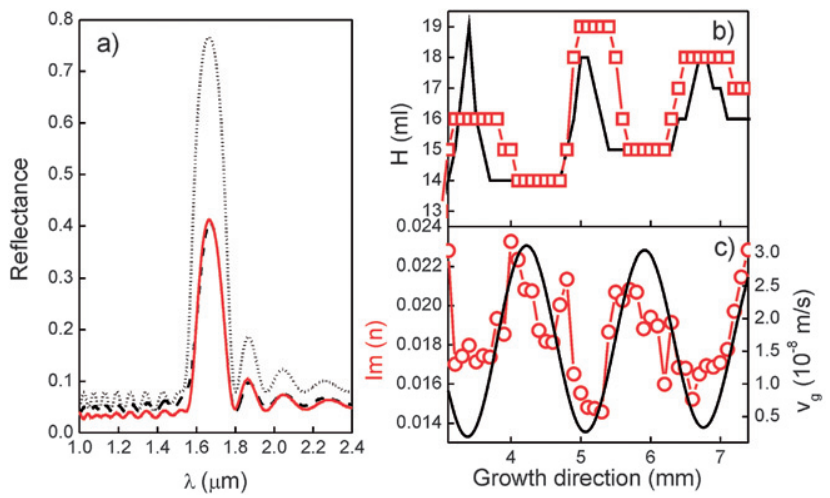

Fig. 2 (a) Measured (red solid line) and calculated specular reflectance spectra (dashed and dotted lines) of a 13-monolayer-thick colloidal photonic crystal film of polystyrene spheres $(n=1.58)$. The dashed line is the optimum fitting attained for $\operatorname{Im}(n)=0.014$ and the dotted line is the reflectance spectra that would result if $\operatorname{Im}(n)=0$. (b) Experimental (colored symbols) and theoretical (black solid line) thickness profile of a colloidal crystals vertically deposited at $35^{\circ} \mathrm{C}$ from polystyrene latex suspensions of $0.2 \%$ particle volume fraction. (c) Time evolution of both crystal growth velocity $v_{\mathrm{g}}$ (black solid line) and the experimentally estimated imaginary part of the refractive index $\operatorname{Im}(n)$ (scatter graphs), extracted from the fitting of the optical reflectance measured from the colloidal crystal analysed in (b).

opening of a stop band between the lowest energy photonic bands along the $\Gamma-\mathrm{L}$ direction in reciprocal space, which corresponds to the [111] direction in the real space face centred cubic structure. Secondary lobes account for the coherent effects arising from the interference of beams reflected at the top and the bottom surfaces of the film, and therefore provides information on its thickness. In Fig. 2(b) we show an example of the experimental and theoretical thickness profiles attained from the fittings of the optical reflectance from different areas of a colloidal crystal film. Apart from the fair fitting of the experimental observations, the model explains several trends that had been empirically described before. $^{18,21}$

The proposed model explains the observed thickness spatial variations, but also yields other relevant structural information. It has been repeatedly shown that, in order to attain an optimum fitting of the optical reflectance like that shown in Fig. 2(a) (dashed line), it is necessary to introduce certain extinction in the structure through an imaginary part of the refractive index of the spheres $\operatorname{Im}(n){ }^{22,23}$ In fact, it can be rigorously demonstrated that losses in the form of diffusely scattered light, introduced, for instance, by imperfections such as particle size dispersion or vacancies, can be approximated by such imaginary part of the refractive index of the spheres for weakly disordered systems. ${ }^{24}$ Thus, in our case $\operatorname{Im}(n)$ is an extinction coefficient directly related to the density of intrinsic defects in the structure. A comparison explicitly shows that there is a strong correlation between the magnitude of $\operatorname{Im}(n)$ required to fit the spectra and the crystalline film growth speed given by eqn (1). Interestingly, those parts of the film that are formed at the lowest speed (thicker regions) present the lowest number of imperfections, while those that are deposited faster (thinner regions) are more defective. Also, the damping of the oscillations seen for $\operatorname{Im}(n)$ and $H$ is also observed for the amplitude of the velocity fluctuations, further confirming their correlation. In Fig. 2(c) we plot the corresponding variation of $\operatorname{Im}(n)$ (related to intrinsic defect density). These features have been repeatedly observed for a large number of samples, hence confirming the relation between film growth speed, crystal thickness and density of intrinsic defects in colloidal crystal films. Other groups have recently reported thorough analyses of the variations of crystal thickness along the growth direction ${ }^{21,25}$ or of the relation between crystal thickness and defect density or domain size, ${ }^{23,26}$ such observations being in fair agreement with those reported in ref. 18 and 19.

A complete picture of the film growth process still lacks a description of the ordering of microspheres in a facecentred-cubic lattice within a receding meniscus. In this regard, a model developed specifically for colloidal crystallization in wet thin films of colloidal suspensions onto non-horizontal substrates has recently been reported, demonstrating the importance of the fluid phase kinematics through the forming particle array. ${ }^{27}$ These recent results will be valuable in the rational design of experiments aiming at achieving perfect lattices. As we remark in the following section, this is the major challenge we face in the field in order to take full advantage of the three-dimensional structure of colloidal lattices, whose main and specific optical fingerprint is a complex photonic response for wavelengths smaller than the lattice parameter.

\section{Effect of imperfections on the optical response at short wavelengths}

The observation of fine optical features at $\lambda \leq a$ ( $a$ being the lattice constant of the conventional cubic cell) was first possible when the method of evaporation induced self-assembly onto vertical substrates was used to form crystals of polystyrene or silica particles of diameters larger than $350 \mathrm{~nm}$. At those spectral ranges, a complex band structure describes the photon modes within a sphere fcc lattice, as illustrated in Fig. 3(a). Above that energy threshold, highlighted by a dashed line in this graph, the reflectance and transmittance spectra of colloidal crystals show a fine peak structure that do not correspond to the presence of any photonic stop bands or blind modes ${ }^{28}$ as has been demonstrated before. Typical specular reflectance and forwards transmittance spectra of a colloidal crystal film are plotted in Figs. 3(b) and 3(c) respectively. Also as a consequence of the improved ordering and structural quality achieved, it was possible to observe visible diffraction for the first time in such colloidal crystal films. ${ }^{3,11}$ In Fig. 4(a), we show a scanning electron microscopy image of the outer (111) plane of a colloidal crystal film along with a picture, displayed in Fig. 4(b), of the corresponding diffraction pattern observed when a laser beam of $\lambda=539 \mathrm{~nm}$ 

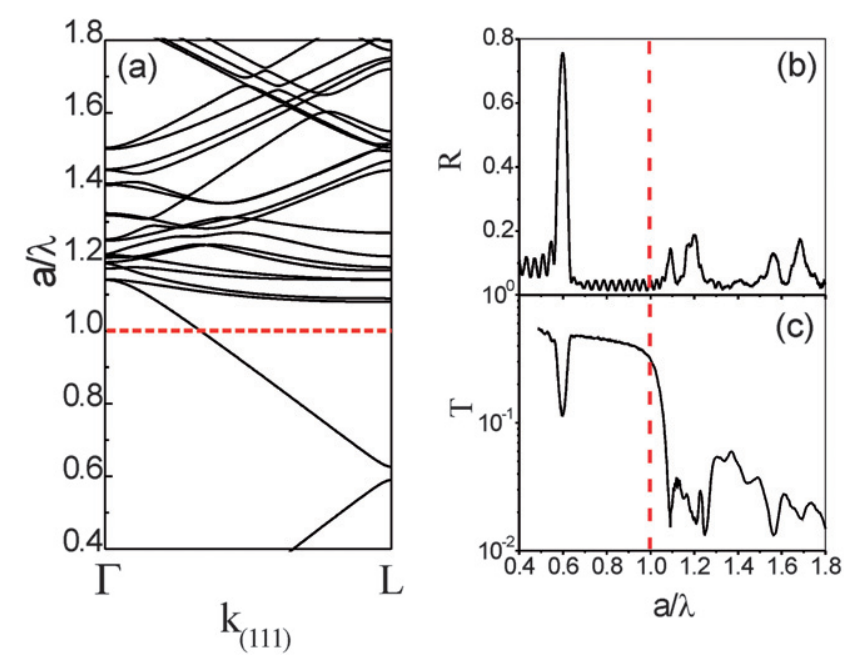

Fig. 3 (a) Calculated photonic band structure along the $\Gamma-\mathrm{L}$ direction of an fcc lattice of polystyrene spheres of refractive index $n=1.58$. (b) Reflectance and (c) transmittance spectra measured from a 18-monolayer-thick colloidal photonic crystal film of polystyrene spheres. Horizontal and vertical dashed lines are plotted at $\lambda=a$.

impinges perpendicular to such planes. A $1 \mathrm{~mm}$ diameter circular spot on the sample was illuminated, hence showing the existence of ordering in the long range in the sample. It can be readily seen that the pattern shown in Fig. 4(b) is the Fourier transform of a triangular arrangement like that observable in
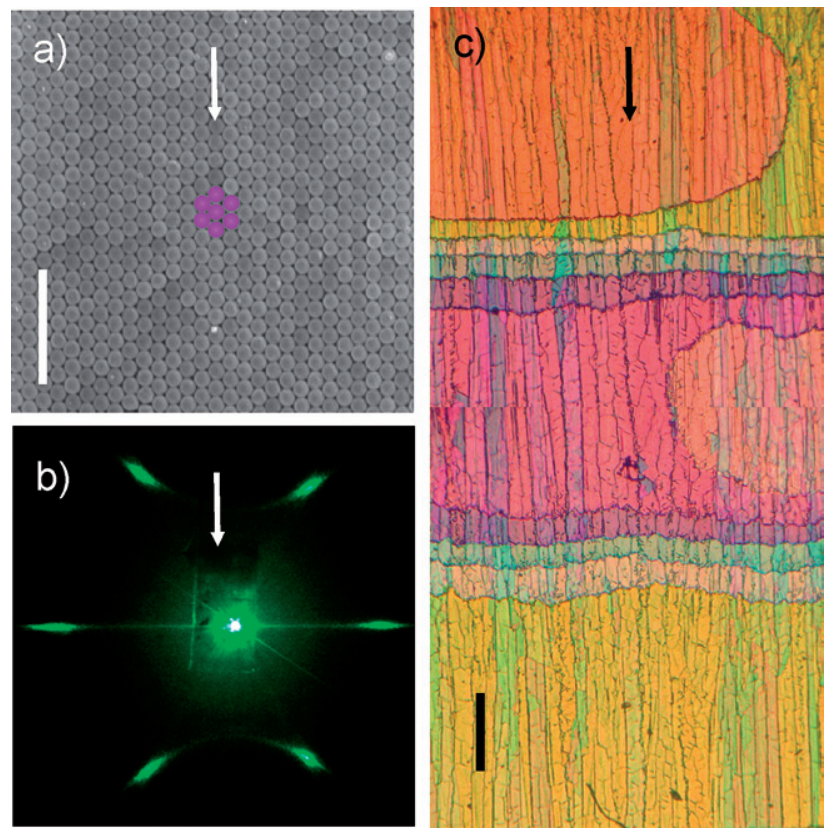

Fig. 4 (a) SEM micrograph of the surface of a polystyrene sphere colloidal crystal. One sphere surrounded by another six are coloured to show the triangular arrangement of the (111) planes. Scale bar is $5 \mu \mathrm{m}$. (b) Diffraction pattern of transmitted beams projected on a screen parallel to surface of the sample for $\lambda=539 \mathrm{~nm}$. (c) Pictures collected along $2 \mathrm{~mm}$ along the growth direction. Areas with the same thickness have been shaded with the same colour. Scale bar is $0.3 \mathrm{~mm}$. Vertical arrows indicate the growth direction in all images. the growth direction of the crystalline film in all three images.

All these optical features occurring at $\lambda$ $\leq a$ were correctly identified as a sign of the improved order achievable with this technique, since in lower structural quality lattices they were barely detectable. The effect of imperfections is actually expected to be much more dramatic when wavelengths become on the order or shorter than the typical size of such defects. Results presented in the previous section demonstrate that such effect must be considered in the calculations in order to accurately reproduce the experimental optical response at wavelengths for which the lower stop bands opens. However, until very recently, the lack of adequate theoretical models to describe the optical properties at $\lambda \leq a$ made it impossible to establish a rigorous comparison between experimental measurements and the response predicted for perfectly ordered structures. This prevented a reliable assessment of the quality of a lattice based on such higher energy response. In a recent report, we have used a modification of the vector Korringa-Khon-Rostoker method ${ }^{29,30}$ to simulate the optical properties of face-centered-cubic structures of spheres at $\lambda \leq a$. This approach allowed us to describe for the first time the optical reflectance and transmittance spectra of a perfect lattice of finite size. Remarkably, a strongly fluctuating reflectance and transmittance is expected for such ideal crystals, a large number of peaks and dips being expected for $\lambda \leq a$. We proved that these features can be rigorously related to multipolar resonances of the sphere ensemble excited by an external incident plane wave. ${ }^{31}$ Using these ideal structures as a starting point, we studied the effect of imperfections on the optical properties by introducing a complex refractive index whose imaginary part $\operatorname{Im}(n)$ was gradually increased. Varying this parameter, an excellent agreement between the simulated and the experimental spectra measured by three different groups was found, an indication of the validity of the above-mentioned theoretical approach. ${ }^{15}$ In Fig. 5(a) we show the expected optical reflectance for an ideal cubic close packed lattice of almost transparent spheres $\left(n_{\mathrm{s}}=1.58+\right.$ $10^{-5}$ i) and in Fig. 5(b) for the structure made of the same spheres but presenting significant optical losses $\left(n_{\mathrm{s}}=1.58+\right.$ 

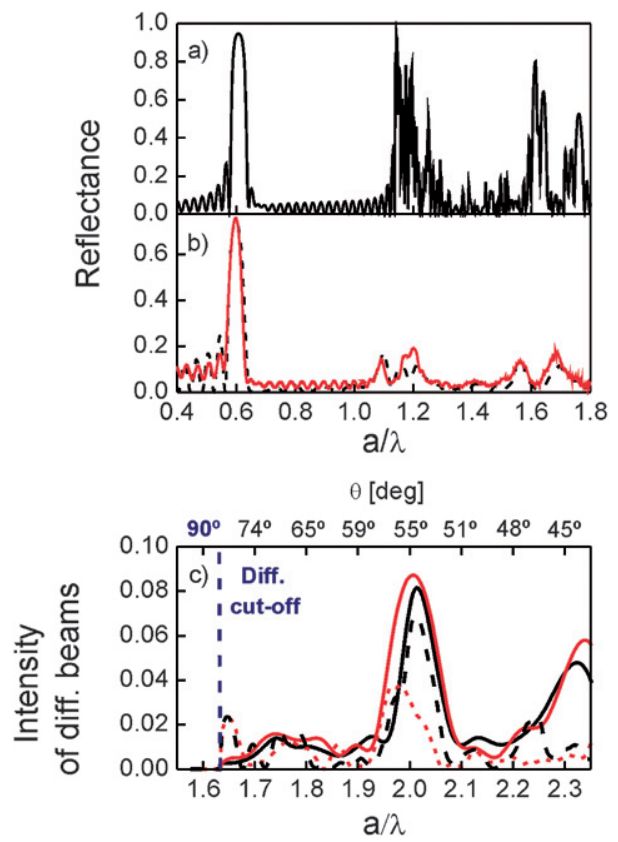

Fig. 5 (a) Calculated specular reflectance spectrum for spheres of dielectric constant $n_{\mathrm{s}}=1.58+$ $10^{-5}$ i. (b) Measured (solid coloured line) reflectance extracted from a 18 monolayer thick colloidal photonic crystal film of polystyrene spheres and the corresponding fitting spectrum (dashed line), attained for $n_{\mathrm{s}}=1.58+0.013 \mathrm{i}$. (c) Measured (solid lines) and calculated (dashed lines, $n_{\mathrm{s}}=1.58+$ 0.01 i) reflection efficiencies of two different diffraction channels $[(1,0)$ (black lines) and $(1,-1)$ (red lines); see ref. 11 for details].

0.013i). An experimental reflectance spectrum is superimposed in this latter case for the sake of comparison. It can be unambiguously concluded that the effect of imperfections was that of smoothing the strong resonances observed in the ideal structure, only a few robust ones surviving in the spectra of the highest quality structures available up to date. Furthermore, similar resonant modulation and smoothing effects were measured and fitted for the beams diffracted by the structure, as shown in Fig. 5(c). ${ }^{11}$ The extraordinary sensitivity of the short wavelength optical response to subtle structural modifications has been used to distinguish between the different variants of the close packed sphere structure, namely, cubic, hexagonal or random, in very thin opal films. ${ }^{32,33}$ It should be noted that, for $\lambda \leq a$, the extinction caused by imperfections makes the response of a real lattice made of 6 compact sphere layers indistinguishable from the response of thicker ones. ${ }^{34}$ However, this is not the case for the lower energy range, for which the effect of extinction is less striking. In this case, the gradual effect of defects on intensity and width of the peak associated to the lower energy stop band can be observed in crystals whose thickness is comprised between a few and several tens of sphere layers. It is clear from this analysis that the magnitude of the extinction coefficient $\operatorname{Im}(n)$ needed to fit the optical response of photonic crystals for $\lambda \leq a$ is an accurate parameter to evaluate the degree of perfection of colloidal photonic crystals.

\section{Conclusions}

In summary, although important advances have been made in the field of colloidal crystal films since the introduction of evaporation-induced selfassembly onto vertical substrates, realization of defect-free colloidal crystal films has still not been achieved. Two main conclusions can be extracted from the results commented in this highlight: first, modelling of the growth dynamics of colloidal crystal films allows one to establish a relation between the different experimental parameters and the structural features observed, which might open a way to improve the quality of the lattice; second, the optical response at $\lambda \leq$ $a$ provides a reliable and precise means of evaluation of the progress towards that goal, as long as adequate theoretical guidance is available. In this framework, a number of challenges that affects different aspects of the modelling of these materials can be identified. Regarding crystal growth, deeper understanding of the mechanism of sphere assembly in an ordered structure within the film is needed in order to design deposition experiments that give rise to a minimum number of intrinsic defects. Also, from the side of the optical characterization, both the shape and the magnitude of the optical effects detected at $\lambda \leq a$ caused by each one of the different types of defects present in the lattice should be modelled and its relative effect weighted. This might help to clarify those defects whose optical effects are more deleterious. Overcoming these challenges may allow the detailed analysis and full technological exploitation of the complex photonic properties that arise from the three-dimensional order existing in colloidal crystal films.

\section{Acknowledgements}

This work has been funded by the Spanish Ministry of Science and Education under grant MAT200503028 and the Argentinian Agencia Nacional de Promoción Científica y Tecnológica. This work has been realized in the framework of a joint Spanish-Argentinian cooperation project CSIC-CONICET (Grant 2005AR0070).

\section{References}

1 P. Jiang, J. F. Bertone, K. S. Hwang and V. L. Colvin, Chem. Mater., 1999, 11, 2132.

2 S. Wong, V. Kitaev and G. A. Ozin, J. Amer. Chem. Soc., 2003, 125, 15589.

3 F. García-Santamaría, J. F. GalisteoLópez, P. V. Braun and C. López, Phys. Rev. B, 2005, 71, 195112.

4 G. von Freymann, S. John, S. Wong, V. Kitaev and G. A. Ozin, Appl. Phys. Lett, 2005, 86, 053108.

5 J. F. Galisteo-Lopez, M. Galli, A. Balestreri, M. Patrini, L. C. Andreani and C. Lopez, Opt. Exp., 2007, 15, 15342.

6 A. Mihi, H. Míguez, I. Rodríguez, S. Rubio and F. Meseguer, Phys. Rev. B, 2005, 71, 125131.

7 F. García-Santamaría, E. C. Nelson and P. Braun, Phys. Rev. B, 2007, 76, 075132.

8 K. Wostyn, Y. Zhao, B. Yee, K. Clays, A. Persoons, G. de Schaetzen and L. Hellemans, J. Chem. Phys., 2003, 118, 10752.

9 Míguez, V. Kitaev and G. Ozin, Appl. Phys. Lett., 2004, 84, 1239.

10 J. F. Galisteo-López and C. López, Phys. Rev. B, 2004, 70, 035108.

11 L. A. Dorado, R. Depine, G. Lozano and H. Míguez, Phys. Rev. B, 2008, 78, 075102. 
12 D. J. Norris, E. G. Arlinghaus, L. L. Meng, R. Heiny and L. E. Scriven, Adv. Mater., 2004, 16, 1393.

13 S. H. Im, M. H. Kim and O. O. Park, Chem. Mater., 2003, 15, 1797.

14 A. Balestreri, L. C. Andreani and M. Agio, Phys. Rev. E, 2006, 74, 036603.

15 L. A. Dorado, R. A. Depine and H. Míguez, Phys. Rev. B, 2007, 75, 241101(R).

16 S. Schutzmann, I. Venditti, P. Prosposito, M. Casalboni and M. V. Russo, Optics Express, 2008, 16, 897.

17 R. V. Nair and R. Vijaya, Phys. Rev. A, 2007, 76, 053805.

18 G. Lozano and H. Míguez, Langmuir, 2007, 23, 9933.

19 G. Lozano and H. Míguez, Appl. Phys. Lett., 2008, 92, 091904.
20 E. Adachi, A. S. Dimitrov and K. Nagayama, Langmuir, 1995, 11, 1057.

21 R. G. Schimmin, A. J. DiMauro and P. V. Braun, Langmuir, 2006, 22, 6507.

22 J. F. Galisteo-López and W. L. Vos, Phys. Rev. E, 2002, 66, 036616.

23 J. F. Galisteo-López, M. Galli, M. Patrini, A. Balestreri, L. C. Andreani and C. Lopez, Phys. Rev. B, 2006, 73, 125103.

24 L. A. Dorado and R. Depine, to be published.

25 L. K. Teh, N. K. Tan, C. C. Wong and S. Li, Appl Phys. A, 2005, 81, 1399.

26 A. Hartsuiker and W. L. Vos, Langmuir, 2008, 24, 4670.

27 D. Gasperino, L. Meng, D. J. Norris and J. J. Derby, J. Crys. Growth, 2008, 310, 131.

28 F. López-Tejeira, T. Ochiai and K. Sakoda, J. Sánchez-Dehesa, Phys. Rev. B, 2002, 65, 195110.
29 N. Stefanou, V. Yannopapas and A. Modinos, Comput. Phys. Commun, 1998, 113, 4977; Comput. Phys. Commun., 2000, 132, 189.

30 N. Stefanou, V. Karathanos and A. Modinos, J. Phys.: Condens. Matter, 1992, 4, 7389

31 L. A. Dorado, R. A. Depine, G. Lozano and H. Míguez, Opt. Exp., 2007, 15, 17754.

32 X. Checoury, S. Enoch, C. López and A. Blanco, App. Phys. Lett., 2007, 90, 161131.

33 E. Vekris, V. Kitaev, D. D. Perovic, J. S. Aitchison and G. A. Ozin, $A d v$. Mater., 2008, 20, 1110.

34 L. A. Dorado, R. A. Depine, G. Lozano and H. Míguez, Phys. Rev. B, 2007, 76, 245103. 